\title{
Editorial
}

\section{Rapid Deployment of Teleophthalmology in the COVID-19 Pandemic}

\author{
Royce W. Chen, MD ${ }^{1}$ Steven E. Brooks, MD ${ }^{1} \quad$ Yocheved S. Kresch, OD, MS ${ }^{1} \quad$ Suzanne Sherman, OD ${ }^{1}$ \\ C. Gustavo De Moraes, MD, PhD, MPH ${ }^{1}$ Dajzsa D. McDaniel, MHA ${ }^{1} \quad$ Lora R. Dagi Glass, MD ${ }^{1}$ \\ Jeffrey M. Liebmann, MD ${ }^{1}$ George A. Cioffi, MD ${ }^{1}$ \\ ${ }^{1}$ Department of Ophthalmology, Columbia University Irving Medical \\ Center, New York, New York \\ J Acad Ophthalmol 2020;12:e61-e62.
}

On Sunday, March 1, 2020, New York City saw its first confirmed case of SARS-CoV-2 (COVID-19). Two weeks later, the city experienced an exponential increase in the number of acutely ill patients and became the epicenter of the pandemic in the United States, and later the world. The health care systems in the city were rapidly overwhelmed by the surge of critically ill COVID-19 patients and it was clear that business as usual was over. All hands were called on deck to deal with the crisis that was unfolding. The Department of Ophthalmology at the Edward S. Harkness Eye Institute and Columbia University Irving Medical Center was called into action as well. Staff redeployments and a rapidly changing safety environment radically altered the physician and technical workforces and the manner in which clinics could operate.

Over the course of several days, in the interest of public safety, as well as to protect our own employees, we scaled back our clinical operations dramatically. We went from seeing several hundred patient visits per day to seeing only those with urgent problems. We incorporated numerous safeguards for our clinics, including the enhanced use of personal protective equipment, extensive sanitization of exam lanes and equipment, cancellation of all elective surgery, limiting clinical operations to two central facilities, and screening patients at the front door for COVID-19 symptoms or fever. In spite of these measures, it was clear that other innovative approaches were necessary. Even in the midst of a surging pandemic, patients continued to need ophthalmic consultation, triage, and importantly, reassurance. To meet these needs we rapidly adopted and implemented telemedicine protocols across all subspecialty divisions, coupled with a triage plan that allowed us to connect with patients in ways that several weeks earlier might have been dismissed as simply an academic interest.

Telemedicine is not a new concept, but it has not been extensively developed or adapted for ophthalmology (i.e., teleophthalmology)., ${ }^{1,2}$ Worldwide experience managing patients using teleophthalmology prior to the pandemic was extremely limited, particularly in developed countries. Furthermore, most of those efforts had been focused on the use of remote testing sites primarily for the purposes of screening.,4 We were confronted by a much different situation, one that relied on live interaction between patients and physicians.

Our recent implementation of a new electronic health record (EHR) system helped tremendously to facilitate our efforts. Embedded within the system is the capability to schedule and conduct secure, Health Insurance Portability and Accountability Act (HIPAA)-compliant audiovisual visits from a mobile device. It allows a provider to capture images, efficiently document findings, and engage in appropriate management and follow-up. Several critical elements, including technical capabilities, patient and physician acceptance, operational workflows, regulatory compliance, and professional liability, were forced to coalesce given the severe practice limitations and social distancing constraints caused by the virus.

On the technical side, an excellent, secure audiovisual platform was embedded within our EHR, and most patients had access to mobile devices or a computer. The use of this platform, however, was unfamiliar to most of the clinicians, and challenging to many patients, particularly the elderly. As an alternative, the emergency relaxation of HIPAA-related regulatory requirements by the state and federal governments made it possible to utilize unsecured, nonpublic facing platforms such as FaceTime, Skype, and Google Hangouts. The audiovisual capabilities of these platforms provided us with a reasonable ability to visually assess the face, lids, conjunctiva, pupils, confrontation fields, extraocular movements, and eye alignment. Ancillary applications (apps) downloaded to, or accessed from, mobile devices or computers made it possible for patients to quantitatively test their near acuity, conduct an Amsler grid test, and even test their central and paracentral visual field. This full array of testing was not necessary in all patients, and could be both time consuming and unreliable if patients had difficulty using the apps, particularly among our elderly and visually

\author{
Address for correspondence \\ Steven E. Brooks, MD, Edward S. \\ Harkness Eye Institute, 635 west \\ $165^{\text {th }}$ St., New York, NY 10032 \\ (e-mail: seb2204@cumc.columbia.edu).
}

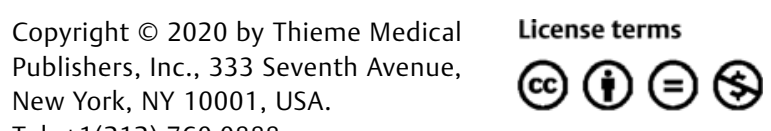

Tel: +1(212) 760-0888. 
impaired patients. In spite of these limitations, the data we gathered was clinically useful, and the potential to improve the testing strategies and modalities was apparent.

To optimize the teleophthalmology workflows, we employed a carefully enhanced triage system of optometrists and ophthalmologists to screen incoming patient calls for urgency. The initial filter was provided by nontechnical staff, who were given training and a triage grid to help determine case urgency. The staff would document the call and chief complaint. If consultation by a provider was considered necessary, they would obtain and document verbal consent for treatment and billing from the patient. The next level of triage included optometrists and ophthalmologists, who could opt for conducting a telemedicine visit immediately or scheduling one at a later date. We found it important to connect with patients prior to the visit and coach them through the telemedicine functions in the EHR. They were also instructed to have a flashlight available and asked to download ancillary apps that might be needed to measure visual function. The parents of pediatric patients were asked to have several small toys and a separate tablet or phone available during the encounter.

In spite of its limitations, patient and physician acceptance was overwhelmingly positive. In the first month we conducted 387 teleophthalmology visits across all subspecialties. In addition to triage and consultative services, the televisits also enabled us to provide much needed reassurance to patients who were dealing with greatly increased levels of anxiety during the COVID-19 crisis. There are specific billing codes that may be used with appropriate modifiers for telephone or teleophthalmology (audiovisual) visits. Images may also be acquired and interpreted, and appropriate professional interpretation codes may be used for this as well.
Spurred by sheer necessity during a global crisis, our rapid implementation of teleophthalmology using easily accessible platforms was instrumental in meeting the needs of our patients at a time when access to conventional care was severely limited. The ability to interact with patients remotely to acquire key clinical data while preserving personal interaction was at the core of this success. Current and future trainees in particular are likely to embrace this technology and may find these sorts of interactions to be a natural extension of social media adapted to ophthalmic care, as well as an opportunity for innovation. Although further investigations are now underway to analyze and validate the quality of the data collected, optimize the examination and scheduling protocols, and determine the most appropriate clinical applications, our early success using teleophthalmology in the midst of the COVID-19 pandemic has been encouraging, and provides an impetus to accelerate the timeline for such work.

\section{Conflict of Interest}

None declared.

\section{References}

1 Sreelatha OK, Ramesh SV. Teleophthalmology: improving patient outcomes? Clin Ophthalmol 2016;10:285-295

2 Available at: https://www.aao.org/practice-management/article/ teleophthalmology-how-to-get-started. Accessed April 28, 2020

3 Kolomeyer AM, Szirth BC, Shahid KS, Pelaez G, Nayak NV, Khouri AS. Software-assisted analysis during ocular health screening. Telemed J E Health 2013;19(01):2-6

4 Shahid K, Kolomeyer AM, Nayak NV, et al. Ocular telehealth screenings in an urban community. Telemed J E Health 2012;18 (02):95-100 